\title{
Aspectos socioeconómicos y percepciones de los corteros de caña sobre la mecanización en Risaralda, Colombia
}

\author{
Socio-economic aspects and perceptions of sugarcane cutters about \\ mechanization in Risaralda, Colombia.
}

\author{
${ }^{1}$ Jhon Jairo Arias Mendoza, ${ }^{1}$ Tito Morales Pinzón, ${ }^{2}$ Andrés \\ Suarez Agudelo \\ ${ }^{l}$ Facultad de Ciencias Ambientales, Grupo de Investigación: Gestión Ambiental Territorial Universidad \\ Tecnológica de Pereira, Pereira, Colombia. \\ tito@utp.edu.co \\ jhonja@utp.edu.co \\ ${ }^{2}$ Administrador Ambiental, integrante del grupo de investigación en gestión y sostenibilidad ambiental \\ GESSA, Universidad de la costa CUC, Barranquilla, Colombia \\ asuarez24@cuc.edu. co
}

\begin{abstract}
Resumen-El artículo presenta la caracterización socioeconómica de los corteros de caña de azúcar del ingenio Risaralda; adicionalmente ilustra las percepciones que los trabajadores tienen con respecto a una transición hacia corte mecanizado, además de su impacto sobre las condiciones de vida. Se realizaron encuestas para identificar algunas características socioeconómicas y dentro de estas, unas palabras claves para determinar las apreciaciones sobre el cambio en la actividad. Los resultados muestran que la mecanización en la recolección de la caña, trae consecuencias significativas en la calidad de vida de los corteros del municipio de la Virginia y del corregimiento de Caimalito.
\end{abstract}

Palabras clave - características socioeconómicas, corteros de caña, ingenio Risaralda, mecanización, percepciones.

\begin{abstract}
This paper shows the socioeconomic characterization of the sugar cane cutters of the Ingenio Risaralda; moreover, the paper illustrates the perceptions of the workers to a transition towards the mechanized cutting of sugar cane and its impact over their livelihoods. Some surveys were used to identify the socioeconomic characteristics and using a number of keywords to identify the perceptions about the technological transition. The results show that cutting mechanized of sugar cane will produce a significant impact on the quality of life not only of La Virginia cutters but also of Caimalito.
\end{abstract}

Key Word - socioeconomic characteristics, sugarcane cutters, Ingenio Risaralda, mechanization, perceptions.

\section{INTRODUCCIÓN}

El manejo tradicional del cultivo de caña de azúcar, involucra la quema de residuos para facilitar la cosecha manual [1]. Lo anterior, para reducir las lesiones a los trabajadores por el fuerte follaje, para combatir insectos y serpientes y para mejorar el rendimiento económico [2]; asimismo se hace para eliminar las hojas con el fin de acelerar la cosecha de la caña de azúcar [3]. Adicionalmente, el facilitar la tarea de cortar genera un aumento en la productividad de los corteros.

Se estima que el $80 \%$ de los cultivos queman la caña para cortarla y el restante $20 \%$ se cosecha en verde [4]. Las quemas se llevan a cabo en Colombia durante todo el año como parte del proceso productivo de la caña de azúcar. Dicha actividad agrícola se extiende por todo el suroccidente del país, a lo largo del valle geográfico del río Cauca, ocupando aproximadamente 200.000 hectáreas, las cuales son sembradas y cortadas con intervalos de 12 meses aproximadamente, es decir, se corta y quema caña de azúcar diariamente [5].

A pesar de todos los beneficios que trae el proceso de quema de la caña, esta es una práctica que genera efectos nocivos sobre el ambiente. La quema de campos de caña antes de la cosecha, genera concentración de monóxido de carbono y emisión de material particulado contribuyendo así al cambio climático; provoca la pérdida de la fertilidad del suelo y genera diversas enfermedades respiratorias [6]. De igual forma, la quema anual de la cosecha de caña de azúcar

Fecha de Recepción: 26 de Abril de 2015

Fecha de Aceptación: 29 de Diciembre 2015 
degrada los ecosistemas y afecta la salud de la población que se encuentra alrededor de los cultivos de caña [7].

Para disminuir los efectos negativos, una de las estrategias consiste en la implementación de maquinaria para la recolección de la caña de azúcar. La mecanización en la recolección, puede resolver los problemas asociados a la quema de la caña de azúcar y reducir la contaminación [3]. La cosecha mecanizada trae grandes beneficios ambientales y en materia de salud al renunciar a la quema de residuos [7]; adicionalmente, proporciona beneficios económicos a los ingenios, ya que reduce el coste de producción del cultivo de la caña de azúcar.

La recolección mecanizada representa una economía hasta del $30 \%$ en relación a los costos por recolección manual en función de un aumento en la productividad, pues la recolección mecanizada puede hacerse hasta por 24 horas ininterrumpidas [7].

No obstante, la mecanización puede disminuir la necesidad de mano de obra ya que una cosechadora es capaz de sustituir de manera eficiente el trabajo manual [6], disminuyendo considerablemente los niveles de empleo en el sector [8]. En este sentido, una sola máquina puede substituir en un día el trabajo de aproximadamente 100 operarios que realizan corte manual [6]; [7]; [9].

En otras palabras, la mecanización del corte de caña puede generar un desplazamiento masivo de trabajadores, debido a que cada máquina cortadora realiza en el mismo tiempo el trabajo de una buena cantidad de corteros.

De manera que la presente investigación busca identificar las formas como los directamente afectados (los corteros) perciben dicha transición (a un sistema mecanizado) y cuál podría ser la repercusión sobre el empleo y las condiciones socioeconómicas en el municipio de La Virginia y en el corregimiento de Caimalito del departamento de Risaralda.

\section{METODOLOGÍA}

\section{A. Área de estudio}

El estudio se llevó cabo en el municipio de La Virginia y el corregimiento de Caimalito del departamento de Risaralda. El municipio de La Virginia se encuentra ubicado a $899 \mathrm{msnm}$ sobre el valle que conforman los ríos Cauca y Risaralda. La temperatura promedio es de 27 grados centígrados y la precipitación en este municipio varía entre 1.800 y 1.900 $\mathrm{mm} / \mathrm{año}$. La Virginia limita al norte con el departamento de Caldas a la altura del municipio de Belalcázar, al sur con el municipio de Pereira (Corregimiento de Caimalito), al oriente con los municipios de Pereira y Marsella, y al occidente con los municipios de Balboa y Santuario. Igualmente el corregimiento de Caimalito presenta las mismas características de temperatura y precipitación (ver Figura 1).

\section{LOCALIZACIÓN DHL ÁREA DE ESTUIDIO}

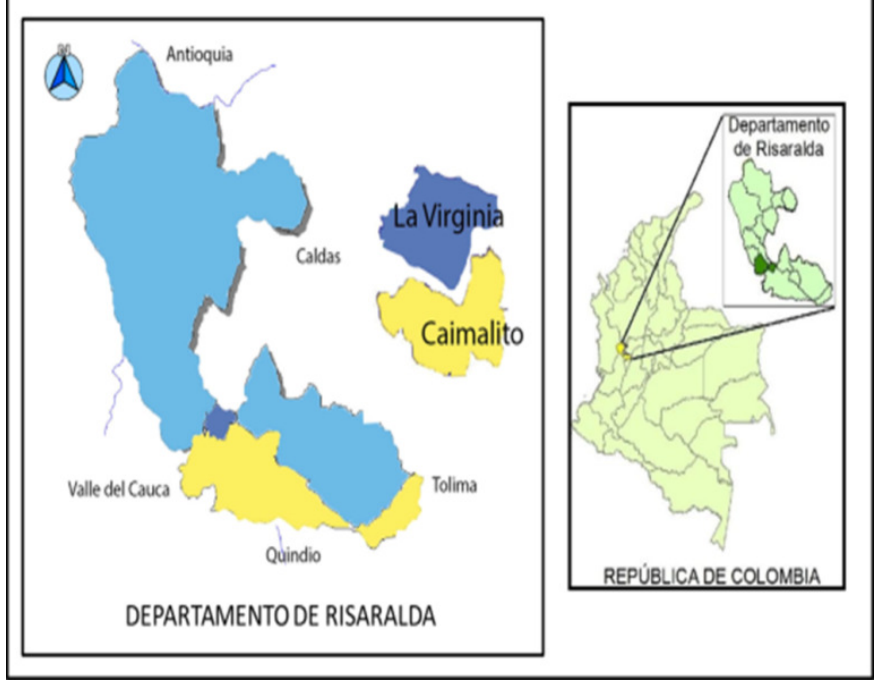

Figura 1. Localización del área de estudio

B. Recolección de información y técnicas de muestreo

La muestra se determinó por medio de un muestreo no probabilístico, mediante la técnica de bola de nieve, debido a la imposibilidad de contar con un marco muestral. La muestra final fue de $n=76$. El proceso de recolección de información se dio a partir del instrumento de la encuesta, donde se indagó sobre las condiciones laborales y características socioeconómicas de los corteros.

Para determinar las percepciones de los corteros con respecto a la transición del corte manual al uso de maquinaria, se generó una serie de palabras claves, a partir de la percepción del proceso de mecanización para la recolección de la caña con relación a las consecuencias asociadas: Desplaza trabajadores, Genera desempleo, Tecnología innecesaria, Mejora la calidad del ambiente, Facilita el trabajo y Mayor tiempo de descanso; conforme a recomendaciones de otros autores [10]. Estas palabras nacen de la revisión bibliográfica y de la interacción con la población objetivo, las cuales ayudaron a la caracterización del problema y representan en un amplio espectro las características y percepciones de los corteros con respecto a dicha transición tecnológica.

Además se realizó un análisis descriptivo y se propuso un modelo que explica las percepciones valoradas en la población encuestada. 


\section{RESULTADOS Y ANÁLISIS}

\section{A. Caracterización socioeconómica de los corteros}

Del total de las encuestas realizadas, el $70 \%$ (52) se realizaron en el corregimiento de Caimalito del municipio de Pereira y el $30 \%$ (24) en La Virginia. Como se observa en la Figura 2, el $56 \%$ de los corteros entrevistados viven en casa propia heredada o familiar y un $44 \%$ paga arriendo.

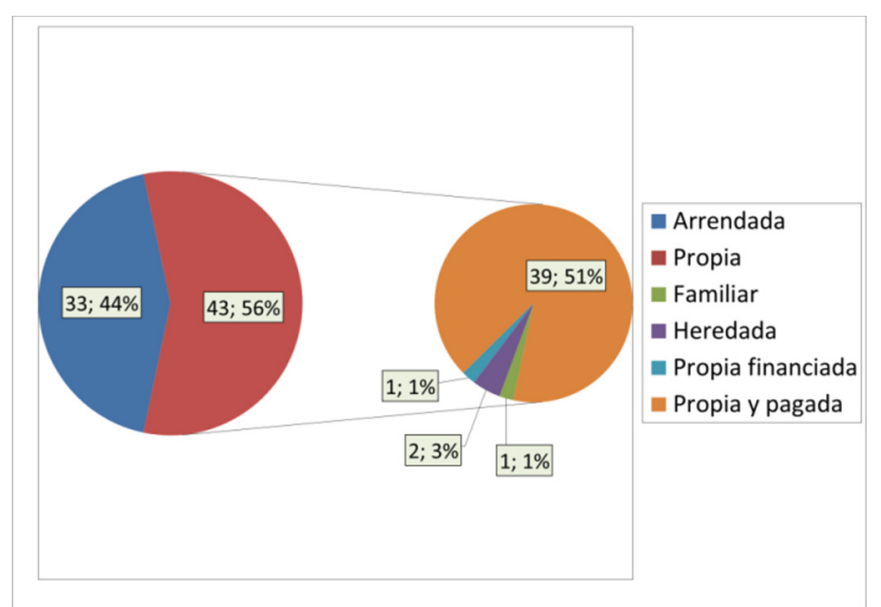

Figura 2. Tenencia de la vivienda de corteros de caña

En cuanto al nivel de escolaridad de los trabajadores que se dedican al corte de la caña, se observa que el $71 \%$ de estos cuentan solo con la primaria, lo que manifiesta altos niveles de vulnerabilidad frente a la posibilidad de quedar cesante en el caso de llevarse a cabo el proceso mecanización del corte de caña (Ver figura 3).

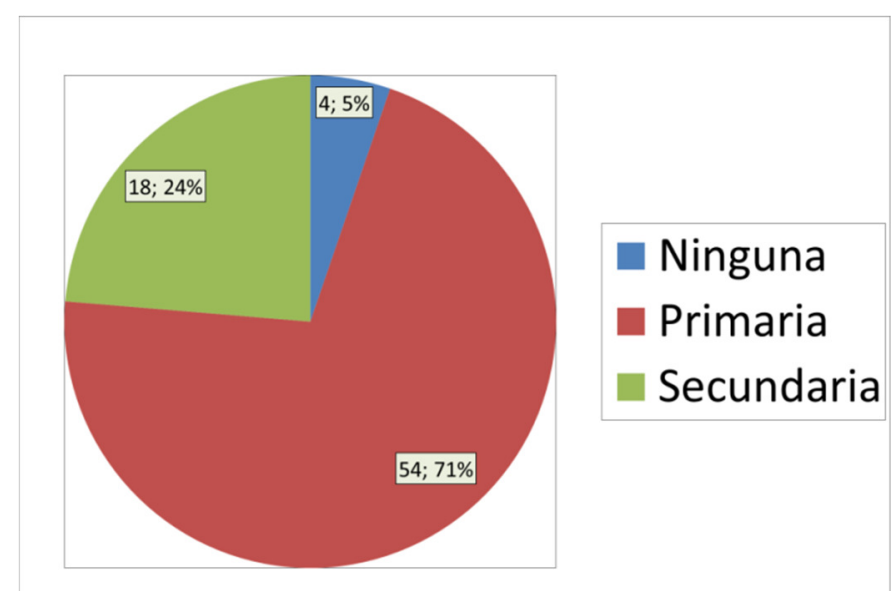

Figura 3. Nivel de escolaridad de los corteros de caña

Existen diferentes modalidades de vinculación laboral de los corteros de caña. De los 76 encuestados, el $74 \%$ de los corteros encuestados están vinculados laboralmente con una cooperativa (Matecaña, Progresemos, Risaralda, etc.), el 22\% están laborando con contratistas que les ofrecen empleo; solo el $3 \%$ trabaja directamente con el ingenio y el $1 \%$ restante aparece como prestador de servicios.

En promedio un trabajador recibe mensualmente US\$387. Sin embargo, 14 encuestados reciben menos de un salario mínimo legal vigente (SMLV=US\$322) y solo 9 pueden alcanzar hasta dos SMLV. El ingreso más frecuente está entre US\$284 y US $\$ 398$ con un total de 30 corteros $(40,5 \%)$ (Ver Figura 4). Frente al tiempo que llevan desempeñándose como corteros, no se encontró una correlación significativa (rho de Spearrman) con el ingreso reportado (rs $>0.05)$, es decir, que no existe evidencia de que el tiempo haya mejorado las condiciones salariales, por lo que la experiencia podría estar siendo subvalorada.

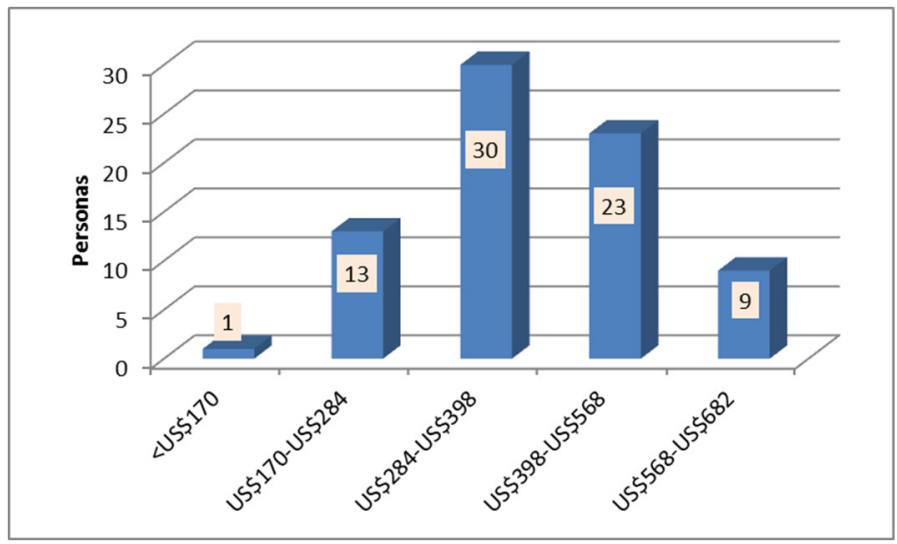

Figura 4. Ingresos mensuales (en dólares americanos) de los corteros de caña

La presencia de familiares que laboran en el mismo sector y actividad es evidente en el estudio. Se encontró que un $61 \%$ de los corteros tiene familiares dedicados a esta actividad, lo cual se puede relacionar directamente con la presencia del Ingenio Risaralda como una de las principales fuentes de empleo en esta región.

Con respecto a la continuidad en el trabajo de corte de la caña a lo largo del año, se encuentra que el $46 \%$ de los corteros entrevistados (35 personas) laboran 12 meses, seguido de un $31 \%$ (24 personas) que lo hacen 8 meses al año y finalmente el $23 \%$ (17 corteros) trabajan en el año por un periodo de 6 meses.

La totalidad de los corteros entrevistados está cobijado por alguna institución prestadora de servicios de salud, entre (ASMED salud, Café salud, Caprecom, Sisben, Comfamiliar, Coomeva, Nueva EPS, Seguro Social, S.O.S, Saludcoop, Salud Total)

Acorde con los resultados arrojados en el proceso de recolección de información socioeconómica, la mayoría de los encuestados aseguró que las condiciones de seguridad laboral son buenas, lo que equivale al $99 \%$ de los encuestados, es decir, (75) corteros; mientras que sólo un $1 \%$ 
de los encuestados equivalente a un cortero expresa que éstas son malas.

La actividad cañera se ha constituido a lo largo del tiempo en actividad fundamental para generar sustento a múltiples familias. Adicionalmente, ha sido un motivo importante para que personas de diversas zonas del país se trasladen a la zona donde se desarrolla esta actividad. Este es el caso del proceso de caña desarrollado por el ingenio, en el cual gran cantidad de personas basan su sustento. En promedio, el número de personas que dependen económicamente del ingreso del cortero es de 3.7 (ver Figura 7), siendo el caso más frecuente el de 3 personas, con una frecuencia de 26 corteros que manifestaron este valor. El total de personas que dependen de la actividad de los 76 corteros encuestados es de 284 personas que viven en la comunidad del corregimiento de Caimalito y en el municipio de La Virginia. Estos datos revelan que una familia integrada en promedio por 4 personas podría estar viviendo con algo más de un SMLV.

Asumiendo que es posible que las características de los encuestados revelen condiciones similares a los 1.196 corteros que se estima existen en la actualidad, se estaría hablando alrededor de 4.425 personas que obtienen el sustento de sus vidas soportados en esta actividad.

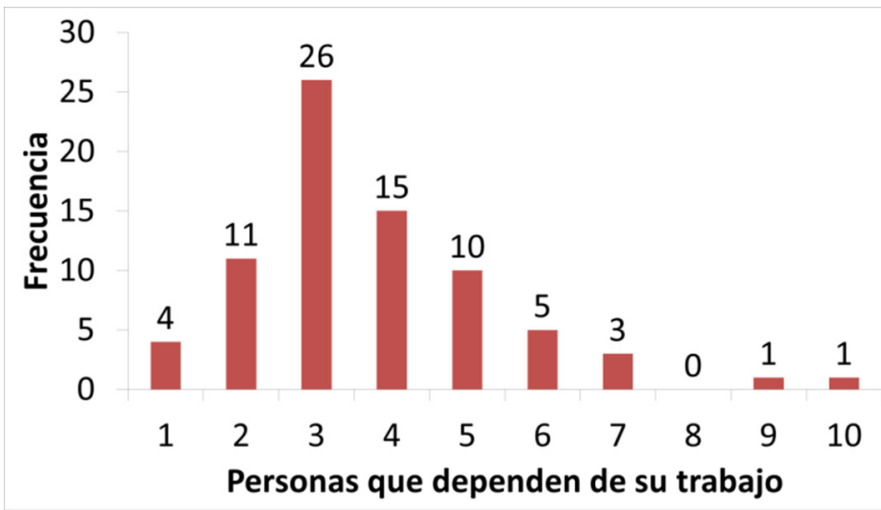

Figura 5. Personas que dependen económicamente del cortero

Un análisis de correspondencias múltiples sobre las variables socioeconómicas, facilitó la comprensión de las características generales de la población encuestada. Así, podemos establecer que los corteros se pueden agrupar en dos grandes grupos: Grupo 1 que corresponde a los corteros que tienen vida familiar estable (casados o unión libre) con mejores condiciones socioeconómicas representadas en mejores ingresos y mejores condiciones de la vivienda; el Grupo 2 se caracteriza por corteros que no tienen dependencia familiar, viven solos, separados o son solteros, incluyen jóvenes con experiencia laboral menor de 5 años y corteros con mucha experiencia laboral (mayor de 20 años) que viven solos (ver Figura 6). Es de resaltar que el Grupo 1 presenta independientemente de la experiencia laboral, mejores condiciones económicas representadas en la posición izquierda del eje horizontal, adicionalmente, la dimensión D2 representa mejor la complementariedad de los ingresos familiares cuando el conyugue labora (generando mayores ingresos para el hogar), situación representada en los puntos localizados en el cuadrante superior izquierdo de la Figura 6.

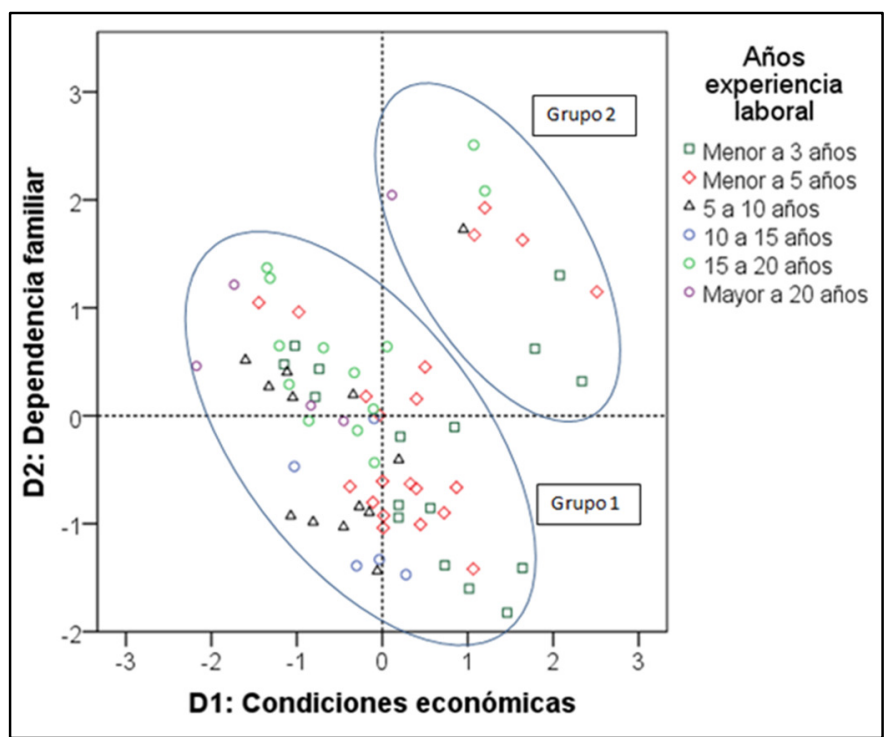

Figura 6. Representación de las variables socioeconómicas derivadas de un análisis de correspondencias múltiples realizado con el software SPSS (versión 15).

B. Transición hacia el corte mecanizado: percepciones e impactos

La evaluación de las percepciones con relación a la transición hacia un proceso mecanizado revela que las palabras seleccionadas fueron adecuadas para resumir las expectativas y temores asociados con la transformación de esta actividad soportada en la fuerza de trabajo de los corteros.

Es evidente que la implementación del mecanizado traería como consecuencia la sustitución de la actividad manual del cortero y eventualmente limitaría las posibilidades de oferta laboral en este sector. Frente a esto es necesario buscar alternativas y otras fuentes de empleo, así como la generación de mayores capacidades en los hogares y en las personas que son cabeza de hogar para poder acceder a otros sectores económicos y estar capacitados para desempeñar otras actividades complementarias.

El análisis de la tabla de frecuencias muestra que existe diferencia significativas (Chi-cuadrado de Pearson <0.05) entre las palabras seleccionadas para describir la transición al proceso de mecanización y la percepción positiva o negativa de la misma (ver Tabla 1). Se encontró que para el grupo de encuestados, las palabras que describen adecuadamente esta transición son: "Desplaza trabajadores" y "Generación de desempleo"; mientras que las palabras que no describen 
adecuadamente son: "Facilita el trabajo" y "Mayor tiempo de descanso".

\begin{tabular}{|c|c|c|}
\hline \multirow{2}{*}{$\begin{array}{l}\text { Palabras asociadas a la } \\
\text { mecanización del cultivo de } \\
\text { caña }\end{array}$} & \multicolumn{2}{|c|}{ Proporción de respuesta } \\
\hline & Adecuado & No adecuado \\
\hline Desplaza trabajadores & $61 \%(5.1)$ & $39,5 \%(-5.1)$ \\
\hline Generación de desempleo & $53 \%(2.0)$ & $47,4 \%(-2.0)$ \\
\hline Tecnología innecesaria & $49 \%(1.3)$ & $51,3 \%(-1.3)$ \\
\hline $\begin{array}{l}\text { Mejora } \quad \text { calidad } \\
\text { ambiente }\end{array}$ & $46 \%(0.9)$ & $53,9 \%(-0.9)$ \\
\hline Facilita el trabajo & $36 \%(-4.6)$ & $64 \%(4.6)$ \\
\hline Mayor tiempo de descanso & $18 \%(-4.6)$ & $81,6 \%(4.6)$ \\
\hline \multicolumn{3}{|c|}{$\begin{array}{l}\text { En paréntesis se muestran los residuos tipificados } \\
\text { corregidos para el análisis de tablas de contingencia. } \\
\text { Todas las medidas direccionales dieron significancia para } \\
\mathrm{p}<0.05 \text {. }\end{array}$} \\
\hline
\end{tabular}

Tabla No. 1 Percepción de la mecanización en proporción de respuestas de las palabras asociadas al proceso

Los resultados de las percepciones muestran una tendencia marcada a considerar el proceso de corte y recolección mecanizada como un proceso negativo. La tabla presenta los resultados de las proporciones en las respuestas dadas por los corteros. Para desplazo de trabajadores, se encuentra que con un $61 \%$ de las respuesta manifestaron que describe de forma adecuada el proceso; por otro lado, otra palabra que se perfila como adecuada es generación de desempleo (53\%). En este orden de ideas, tecnología innecesaria con un $51 \%$ se consideró como no adecuada; de esta misma manera fueron calificadas no adecuada las preguntas mejora la calidad del ambiente (53\%), facilita el trabajo (64\%) y finalmente, genera mayor tiempo de descanso $(81 \%)$.

El foco de atención está dado en lo tocante al empleo, por lo cual no se evidencian otros tipos de beneficios que el proceso podría generar; por ejemplo, disminuir el impacto ambiental. De manera que es ampliamente reconocido que la implementación de maquinaria para desarrollar las labores de corte y recolección reemplazaría el trabajo de muchos operarios. Esta es una realidad bien conocida por los corteros y la visión de ellos queda plasmada en los resultados generados por las palabras clave.

\section{Desplazo de trabajadores y generación de desempleo}

La mecanización en la recolección de la caña, trae consigo consecuencias significativas en la calidad de vida de los corteros tanto del municipio de la Virginia como del corregimiento de Caimalito. Algunas de las implicaciones están relacionadas con la disminución en la oferta de empleo, si se tiene en cuenta que una maquina tiene la capacidad de sustituir alrededor de 100 trabajadores.

No obstante, los efectos no terminarían allí, debido a que los corteros no serían los únicos perjudicados si se tiene presente sus familias. De acuerdo a los datos arrojados por las encuestas realizadas en la zona, los ingresos de los hogares encuestados provienen en su mayoría del salario como cortero (ver Figura 2).

Asimismo, la economía de la región se vería seriamente impactada pues la capacidad de consumo y de empleo de la población disminuiría notablemente. La pobreza, en este caso relacionada con la ausencia o disminución de ingresos, también traería secuelas en el campo de salud ya que probablemente las familias de los trabajadores despedidos, no podrían acceder a este tipo servicios con los que cuentan actualmente, gracias a la afiliación de la cooperativa Progresemos y Procaña, Cooperativa Matecaña, las cuales son las más representativas en este sector.

La Tabla 2, ilustra un escenario sobre las implicaciones que traería la mecanización en la recolección de la caña a diferentes porcentajes sobre el total de los trabajadores que realizan el corte y la recolección de la caña. Teniendo en cuenta lo que se menciona anteriormente que 1 maquina puede desplazar hasta 120 trabajadores aproximadamente.

\begin{tabular}{ll}
\hline $\begin{array}{l}\text { Mecanización } \\
\text { desplazados* }\end{array}$ & $\begin{array}{l}\text { Corteros caña } \\
\text { de }\end{array}$ \\
\hline $25 \%$ & 120 \\
$50 \%$ & 299 \\
$100 \%$ & 598 \\
& 1196
\end{tabular}

*Calculado sobre la base del total de trabajadores existentes.

Tabla 2. Sustitución esperada de trabajadores según porcentajes $d e$ mecanización.

Por lo tanto, si hubiese una transición de corte manual de caña a una mecanización del 10\%, habría 120 corteros de caña desplazados por este proceso. Como el total de trabajadores en este sector es de 1196 trabajadores, para sustituirlos completamente se requieren aproximadamente 10 máquinas. Es de aclarar que estas máquinas estarías operadas por un tipo de trabajador calificado seguramente diferente al cortero. 


\section{CONCLUSIONES}

Si se realiza el proceso de transición, éste debería estar acompañado de dinámicas que propendan por asegurar el sustento a los corteros y familias que de él dependen, que se vean afectados por el cambio en la actividad de corte. Lo anterior, a partir de programas de reinserción laboral o de realizar la transición pero con acompañamiento activo de los corteros.

De los análisis elaborados en el estudio se puede afirmar que debido a la vulnerabilidad de las personas que dependen de esta actividad, la mecanización, a futuro se podría desencadenar problemáticas sociales que afectarían directamente a la comunidad, y en particular, el desarrollo económico de un municipio que presenta poca oferta laboral siendo el principal empleador el sector cañero.

El desarrollo de dinámicas que propendan directamente por la conservación ambiental, no siempre es una solución elocuente a las problemáticas presentes en una comunidad, ya que en términos ambientales se debe buscar un equilibrio tanto del hombre en el medio y generar alternativas para la armonización de estas actividades a futuro sin una afectación directa sobre las condiciones que ofrecen oportunidades de empleo a comunidades necesitadas.

Las percepciones que manifiestan los corteros de caña sobre el proceso de transición, son tendientes a ver el proceso como algo negativo y que no debería ser. Lo anterior, debido a que se reconocen las implicaciones que traería sobre el bienestar de la población, la implementación del corte mecanizado aunque pueda tener algún beneficio en términos ambientales.

\section{AGRADECIMIENTOS}

Los autores agradecemos a Katherine Pardo Celis Eliana Marcela Salazar, Oscar Alejandro Montoya Trujillo Verónica Mafla Saldarriaga, Catalina Bravo Salazar, Lina Marcela Saldarriaga Carmona, Mary Luz Trejos López y a Vanessa Mejía miembros del semillero de investigación en Economía Ambiental y de los Recursos Naturales, perteneciente al Grupo de Investigación en Gestión Ambiental Territorial quienes participaron en el proyecto. También agradecemos a la Universidad Tecnológica de Pereira por haber brindado la financiación el proyecto "Implicaciones de la transición del corte manual de caña a la mecanización en el municipio de la Virginia y el corregimiento de Caimalito (Pereira)", proyecto que dio origen a este artículo.

\section{REFERENCIAS}

[1]. Panoso, A et al., (2011). Soil $\mathrm{CO}_{2}$ emission and its relation to soil properties in sugarcane areas under Slash-and-burn and Green harvest. Soil and Tillage Research. Vol.111, pp. 190-196.
[2]. Allen, A et al., (2004). Influence of sugar cane burning on aerosol soluble ion composition in Southeastern Brazil. Atmospheric Environment, vol. 38, pp. 5025-5038.

[3]. Cansee, S. (2010). A Study of Sugarcane LeafRemoval Machinery during Harvest. American Journal of Engineering and Applied Sciences, vol. 3, pp.186-188.

[4]. Espinal et al., (2005). La cadena del azúcar en Colombia: Una mirada global de su estructura y dinámica 1991-2005. Documento de trabajo número 56. Ministerio de Agricultura y Desarrollo Rural, observatorio de agrocadenas Colombia.

[5]. Dávalos, E. (2007). La caña de azúcar: ¿una amarga externalidad?. Desarrollo y Sociedad. Vol. 59, pp.117-164.

[6]. Shikida, P et al., (2007). Mudanças no padrão tecnológico do corte de cana-de-açúcar: Umaanálise preliminar do caso Paranaense. Umaanálise de cenário. Revista de ciênciasempresariais da UNIPAR, vol. 8, pp. 7-32.

[7]. Junqueira, $P$ et al., (2009). Mudança institucional e o impacto no padrão tecnológico: o caso da mecanização da colheita de cana-de-açúcar no Paraná. Vol. 11, pp. 87-105.

[8]. Guilhoto, J et al., (n.d). Mechanization Process of the Sugar Cane Harvest and Its Direct and Indirect Impact over the Employment in Brazil and in Its 5 Macro Regions. Consultado el 5 abril, 2011. Disponible en:

[9]. http://www.usp.br/feaecon/media/livros/file_213.pdf

[10]. Plec, O et al., (2004). Mecanização do corte da cana-de-açúcar como fator de sustentabilidade ambiental no Paraná: Umaanálise de cenário. Revista De Ciências Empresariais Da UNIPAR, vol. 8, pp. 53-72.

[11]. Rye, J. (2006). Rural youths' images of the rural. Journal of Rural Studies, vol. 22, pp. 409-421. 